\title{
MEDIA EDUCATION IN LATIN AMERICA (2019)
}

\section{Julio-César Mateus, Pablo Andrada y María-Teresa Quiroz (Eds.). Oxfordshire: Routledge}

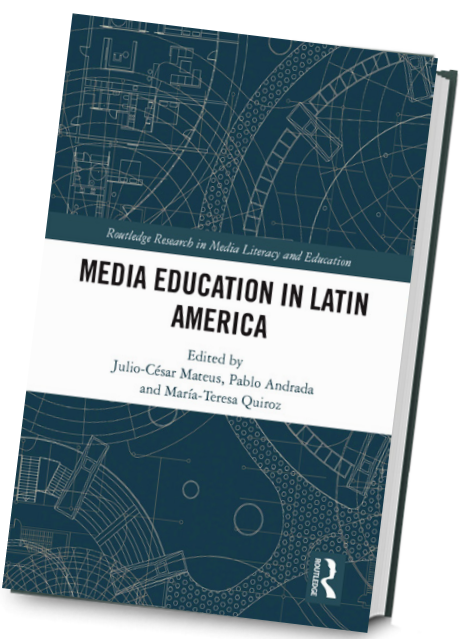

Por Diana Rivera-ROgé ${ }^{1}$

Universidad Técnica Particular de Loja (Ecuador) drdianis@gmail.com

Media Education in Latin America constituye una obra de gran magnitud, publicada por la editorial Routledge, que trasciende a la visión tradicional de la relación entre los medios y la educación gracias a la labor de sus editores.

Este libro aporta una visión global de la educación en medios en América Latina, comenzando, a manera de introducción, con un breve repaso por el contexto de la relación entre los medios de comunicación y la educación en países como Argentina, Bolivia, Brasil, Chile, Colombia, Ecuador, El Salvador, México, Perú, Uruguay y Venezuela, haciendo énfasis en sus propuestas educativas en el entorno de sus contextos políticos, marcos regulatorios, actores sociales, profesorado, producción académica en temas de educomunicación y desafíos de la educación en medios en la región.

Lo anteriormente señalado constituye una herramienta clave en la configuración de la alfabetización mediática en cada uno de los países frente al desarrollo de nuevas propuestas y estrategias que permitan impulsar la educación relacionada con el uso y consumo de medios de comunicación, como uno de sus pilares clave.

Finalmente, se incluye una serie de ensayos críticos que permiten conocer los hitos en América Latina a lo largo de los últimos 50 años, junto con una propuesta de un nuevo

1 Doctora en Comunicación por la Universidad Santiago de Compostela, España. https://orcid.org/00000001-8965-0170 
concepto de alfabetización, recepción constructivista de la televisión en los niños, alfabetización mediática en el aprendizaje contemporáneo y los nuevos desafíos de la educación, este último tema se enlaza con la alfabetización transmedia y culturas participativas.

En la primera parte, adoptando un enfoque temático y basado en cada país, los investigadores presentan una perspectiva integral de América Latina, en la que abordan los contextos políticos y sociales en los que se basa la educación en medios, el estado actual de las políticas educativas con respecto a los medios, organizaciones y experiencias que promueven la educación en medios. En ese contexto, se conoce que, en la región, a medida que el gasto en infraestructura tecnológica aumenta con fines educativos, los planes de estudio nacionales comienzan a implementar habilidades relacionadas con los medios.

Esta parte está compuesta por 12 capítulos. En el primero de ellos: "Educación en medios en Argentina. balance y perspectivas de un campo en construcción", sus autoras, Bettina Martino y Silvana lovanna Caissón, explican el proceso de transición de la educación en medios de Argentina. Este proceso no ha estado absuelto de regulaciones y prácticas socioculturales diversas, acompañadas por el surgimiento de novedades en la preparación y ejercicio profesional en torno a la educación y a los medios que coadyuvaron a la generación de nuevas experiencias y prácticas en escuelas y organizaciones.

En el segundo capítulo, "Educación en medios en Bolivia. Algunos avances y la necesidad de propuestas integrales", el investigador René Zeballos, realiza un análisis general de Bolivia enfatizando en los limitados avances de esta nación con respecto a políticas, estrategias y acciones en el campo de competencia y educación en medios, especialmente para niños, adolescentes y jóvenes. Se incluye en el currículo de primaria y secundaria secciones de comunicación humana, de masas y digital; sin embargo, su diseño muestra que todavía existe una debilidad frente a la integralidad entre los procesos de enseñanza-aprendizaje y metodologías y hace falta poner énfasis en la "comprensión crítica".

El tercer capítulo, "Educación en medios en Brasil. Dilemas, límites y posibilidades", de Mónica Fantin, buscaba situar algunos dilemas, límites y posibilidades relacionados con la educación en medios. En este sentido, la autora menciona que la educación mediática en este país puede contribuir a prácticas disruptivas y formas de resistencia que ahora están tomando forma en Brasil.

"Educación en medios en Chile. Un salto digital que abandonó el estudio de medios" se titula el cuarto capítulo, de la autoría de Pablo Andrada, Cristian Cabalín y Rayén Condeza. Ahí relatan que la educación en Chile está en un momento clave, se ha avanzado en aumento de cobertura de la educación y mayor acceso a tecnologías, las autoridades han privilegiado sus inversiones en computadoras y conectividad para escuelas a través del programa Enlaces. 
El quinto capítulo, del investigador Diego Leandro Marín Ossa, titulado "Educación en medios en Colombia. Una herencia con posibilidades y desafíos para el siglo XXI", permite conocer la realidad de la educación mediática en Colombia. Hace hincapié en una serie de recomendaciones en el marco regulatorio, instituciones y actores sociales, y en cuanto a formación del profesorado, a partir de estos cambios el autor sostiene que pueden llevarse a cabo a nivel individual y colectivo procesos de educación mediática.

En el capítulo seis, "Educación en medios en Ecuador. Exploración y descripción de una necesidad latente", de las investigadoras Catalina González Cabrera y Cecilia Ugalde, se revela que el nivel de alfabetización mediática en estudiantes, profesores y padres de familia de instituciones educativas públicas y privadas es medio. Por otro lado, la capacitación ofrecida por el gobierno es principalmente limitada a la introducción de las TIC y al uso básico de algunas de sus herramientas, el reto pendiente es la orientación hacia la alfabetización mediática. Las autoras concluyen que, a pesar de los muchos problemas, existen condiciones favorables para la integración de la educación en medios en la realidad del sistema escolar ecuatoriano.

“Educación en medios en El Salvador. Ritmo lento en el camino hacia la alfabetización mediática" se constituye en el séptimo capítulo de la autoría de Amparo Marroquín Parducci, Willian Carballo y Nelly Chévez. Los investigadores llegan a la conclusión de que este país cuenta con un alto grado de consumo de medios, pero sin la educación necesaria para avanzar y convertirse en una audiencia que disfruta de contenidos desde una perspectiva informada y crítica (alfabetización mediática). Esto se debe principalmente a dos factores; por un lado, hay poca preocupación por los procesos de comunicación $y$, por otro lado, ciertos intereses políticos sobre la mesa que no apoyan los procesos educativos de la sociedad.

En el capítulo ocho, "Educación en medios en México. Por la formación de una ciudadanía crítica", los autores Julieta Flores Michel, Alma Elena Gutiérrez Leyton y Rosario Lucero Cavazos Salazar nos permiten conocer que las acciones para promover una alfabetización mediática efectiva en México se han fortalecido con la intervención de la televisión cultural, las ONG y la Secretaría de Educación Pública. No obstante, el establecimiento de las políticas en este sentido se está ejecutando a través de la implementación de equipos tecnológicos, por ejemplo, se han entregado tabletas o impresoras 3D a instituciones educativas, pero, al igual que en el resto de países de Latinoamérica, hace falta un proceso de alfabetización.

"Educación en medios en Perú. Un campo lleno de oportunidades", de Ana-María CanoCorrea y Rosario Nájar-Ortega, constituye el noveno capítulo. Las autoras ofrecen una visión de las iniciativas peruanas para mejorar la calidad de la educación y las relacionan con las experiencias puestas en marcha a través del aparato estatal. En ese contexto, 
se considera que existen aún pocos avances en la educación en medios y una incipiente gestión de iniciativas y políticas públicas sobre el tema. Esto ha llevado a que proyectos relevantes pierdan continuidad y se conviertan en experiencias aisladas y desarticuladas.

Siguiendo con las experiencias de los países de América Latina, los autores Rosario Sánchez Vilela, María Lucía Gadea y María Laura Rocha abordan el capítulo 10: “Educación en medios en Uruguay. Entre una brecha digital estrecha y la persistencia de una brecha educativa". Los autores nos permiten conocer que Uruguay presenta ciertas características que lo convierten en un caso especial en Latinoamérica. Uno de estos aspectos es que ha alcanzado importantes niveles de equidad en la distribución del ingreso, pero mantiene enormes desigualdades con respecto al acceso al conocimiento, al mismo tiempo, que posee una brecha digital estrecha. Así, el tema de la educación en medios aparece en la legislación y es parte de los programas de educación primaria y secundaria como tema sugerido o estrategia didáctica, pero no se ha implementado en el currículo, salvo algunos talleres como espacios extracurriculares.

"Educación en medios en Venezuela. De frenesí a contradicciones", de Morella Alvarado Miquilena, Alexandra Ranzolin y Cristina Méndez Pardo, se constituye en el último capítulo de esta primera parte. Nos permiten conocer que la situación de la educación en medios en Venezuela no carece de propuestas por los diversos actores, pero sufre la desarticulación entre quienes deberían elaborar acuerdos para integrar las diversas iniciativas a través de políticas públicas coherentes, sistemáticas e inclusivas, que permitan proporcionar estrategias financieras sólidas, sobre todo, enmarcadas en los principios de libertad y autonomía. Por lo tanto, es fundamental implementar un programa integral de educación en medios.

La segunda parte de la obra se compone de ensayos críticos, en los cuales sus autores reflexionan acerca de los cambios, avances y perspectivas de futuro de la educomunicación.

El primer ensayo está a cargo del maestro Ismar de Oliveira Soares, "Hitos de la educomunicación en América Latina: ¿qué debe considerarse en los últimos 50 años?". Allí, hace un recuento de la educación en medios en los años 60 y 70, el enfoque ideológico, comunicación para el desarrollo social, algunos programas de educación mediática e investigaciones en este ámbito para cerrar su aporte con la educomunicación en la era digital.

El ensayo "Hacia un nuevo concepto de alfabetización", de Roxana Morduchowicz, hace una revisión y reflexión bibliográfica de los principales autores que abordan el tema, con el firme propósito de contar con un nuevo concepto de alfabetización informativa del siglo xxı, que permita a los ciudadanos analizar, procesar, comparar, cuestionar, evaluar, decidir y participar. 
El tercer ensayo se denomina "TV constructivista. Recepción en los niños de salón de clases", de la autoría de Valerio Fuenzalida, quien da a conocer la evolución de educación televisiva y los esfuerzos que se están realizando para producir "Edutainment" dirigido a la inteligencia emocional. Así, el autor manifiesta que la educación preescolar ofrece una valiosa oportunidad para adoptar un modelo constructivista de recepción de TV en el aula.

El ensayo cuatro, de la firma de los profesores Guillermo Orozco Gómez y José Manuel Corona Rodríguez, "Alfabetización mediática basada en el aprendizaje de las sociedades contemporáneas", aborda el tema de la sociedad de la información, nuevas formas de aprendizaje autónomo, intensivo, mediado y colectivo, y la alfabetización mediática como una necesidad en la sociedad del aprendizaje.

Con el tema "Revisión crítica del sentido crítico", Joan Ferrés realiza una revisión de las dimensiones del pensamiento y conciencia crítica, de la información y los mensajes, colocando algunas experiencias para expandir el sentido crítico.

Para finalizar, "Alfabetización transmedia y culturas participativas. Una agenda de investigación", de Carlos Alberto Scolari, reflexiona sobre la necesidad de que la alfabetización transmedia sea parte del nexo entre la juventud, los medios y la educación, desde una perspectiva distinta a la victimización de los niños frente a la televisión y que, por el contrario, englobe el desarrollo de las relaciones individuales y sociales en espacios mediatizados, como otro entorno de interacción.

La lectura de esta obra permitirá el desarrollo de una visión sistemática de la educación en medios en América Latina, al tiempo de enriquecer el debate sobre la importancia y necesidad de la alfabetización mediática como un derecho de los ciudadanos.

\section{REFERENCIAS}

Mateus, J.-C., Andrada, P. y Quiroz, M.-T. (Eds.). (2019). Media Education in Latin America. Oxford: Routledge. 
